\title{
$\&$ Vascular injury during spinal procedures
}

\author{
Robert F. Heary, MD, ${ }^{1}$ and Praveen V. Mummaneni, MD² \\ ${ }^{1}$ Department of Neurological Surgery, Rutgers New Jersey Medical School, Newark, New Jersey; and ${ }^{2}$ Department of \\ Neurosurgery, University of California, San Francisco, California
}

$\mathrm{A}$ RIYOSHI et al. have presented an extremely important paper because it addresses a commonly performed spine surgical procedure that could have resulted in a fatal complication. ${ }^{1}$ There are an assortment of items to be gleaned from this paper that will be useful to all spine surgeons who perform posterior approach interbody fusions (either posterior lumbar interbody fusion [PLIF] or transforaminal lumbar interbody fusion [TLIF]).

A brief synopsis shows a 74-year-old woman with severe lumbar stenosis/scoliosis who was a candidate for a decompression procedure with deformity correction. The preoperative images demonstrate the stenosis as well as the severe coronal plane scoliosis with both translation and rotation at the L2-4 levels. A posterior-alone multilevel TLIF was performed. During this operation a cage was lost through a defect in the anterior anulus fibrosus and the anterior longitudinal ligament, and the cage ended up in the retroperitoneal space between the aorta and the inferior vena cava (IVC). Because no appreciable bleeding was identified, the surgeons chose to finish the case and retrieve the anteriorly misplaced cage at a later date.

One week following the index surgery, the cage was removed during an anterior-alone approach. During the surgery, massive bleeding occurred due to injuries to the IVC and both common iliac veins. A vascular surgeon was brought in for this emergency; however, the major venous structures had to be sacrificed because the bleeding had become life threatening. There was a $20-\mathrm{L}$ blood loss requiring massive transfusions of packed red blood cells, fresh frozen plasma, and platelets, along with use of vasopressors. With this type of bleeding in an elderly woman, this was clearly a dire circumstance and the vascular surgeon had to ligate the IVC below the renal veins to obtain hemostasis.

The patient had a satisfactory outcome despite a 12-day stay under intensive treatment. Substantial bilateral lowerextremity edema occurred but the neurological, radiological, and functional outcomes appeared to be quite good.
There are an assortment of issues that merit consideration in the attempt to avoid future similar cases. The first issue involves the use of the term "migration." This term is typically used for an initially properly placed implant that moves at a later time. In this case, the authors were aware of the cage "misplacement" at the time of the index surgery. This technical error could have been avoided by not releasing the cage inserter until a fluoroscopic image was obtained.

The second issue involves the indication for the second surgery in an elderly woman without clear evidence that a vessel had been violated. Pushing a blunt cage against major vessels might very easily not violate the vessel proper. In fact, no major bleeding was detected during the index surgery. If an angiogram and a venogram had been obtained, this might have allowed the second procedure to not be performed at all if no vessel violation was demonstrable.

The third issue that occurred was the failure to have a vascular surgeon in the operating room during the exposure of the vessels and the cage. It is possible that if a vascular surgeon with the skills needed to manipulate the vessels had been involved at the start of the case, the treatment of any injured major vessels might have had a different result.

A similar tragic case was reported in New Jersey, where a minimally invasive lateral approach to the lumbar spine caused multiple major venous injuries, which ultimately resulted in the patient's death 5 weeks after the index procedure. In that case, the surgery was performed in an outpatient same-day surgery center where no general or vascular surgery support was available. The patient was transferred as an emergency case to an acute care hospital with an open, packed, surgical wound; numerous infections followed in the retroperitoneal space requiring weekly reoperations, which eventually failed. ${ }^{2}$

The case presented by Ariyoshi et al. was potentially catastrophic. ${ }^{1}$ Issues have been identified with the index 
surgery, the workup/indications for the second surgery, and the performance of the second surgery. Although the long-term outcome appears to have been satisfactory, this case demonstrates a variety of teaching points to be considered during the execution of a commonly performed fusion surgery.

http://thejns.org/doi/abs/10.3171/2015.7.SPINE15688

\section{References}

1. Ariyoshi D, Sano S, Kawamura N: Inferior vena cava injury caused by an anteriorly migrated cage resulting in ligation: case report. J Neurosurg Spine [epub ahead of print December 4, 2015. DOI: 10.3171/2015.6.SPINE1544]

2. Assina R, Majmundar NJ, Herschman Y, Heary RF: First report of major vascular injury due to lateral transpsoas approach leading to fatality. J Neurosurg Spine 21:794-798, 2014

\section{Disclosures}

Dr. Mummaneni has the following disclosures. AO Spinegrant and honoraria; DePuy Spine-honoraria and royalty; Thieme Publishing, Springer Publishing, and Taylor and Francis Publishing - royalty; Spinicity/ISD — direct stock ownership; and Globus - honoraria.

\section{Response}

\section{Dai Ariyoshi, MD, Shigeo Sano, MD, PhD, and Naohiro Kawamura, MD, PhD}

Department of Orthopaedic Surgery, Sanraku Hospital, Tokyo, Japan

We appreciate your understanding of the importance of our report and that you have given us an opportunity to share the knowledge and information on this type of complication with many spine surgeons. The comments of Drs. Heary and Mummaneni have greatly made up for our deficiencies in discussion and have highlighted additional points to be learned from this experience.

As for the first issue, we have used the term "migra- tion" to describe the situation where the cage was pushed too deeply beyond the anterior anulus into the retroperitoneal space during adjustment of the depth. As the editorial writers pointed out, however, "misplacement" would better express the situation. In addition, we should not have released the cage inserter until a fluoroscopic image or intraoperative plain radiograph was obtained, when we were not able to tell the depth of the cage, considering the possibility of misplacement.

As Drs. Heary and Mummaneni have pointed out, the second issue was that our indication for the second surgery was not clear. Because there was no apparent vascular injury after the initial surgery, leaving the cage alone could have been one of the options. We were afraid that the misplaced cage might cause some problems later, and tried to remove it. However, we should have clarified the reason and considered the risk versus benefit carefully before deciding to operate to remove the misplaced cage.

Unfortunately, we realized the importance of the third issue just when we were performing the operation, and this was a lack of preparation for the possible risks. It is essential to obtain a vascular surgeon's support if there is any possibility of a major vascular injury. After the present case, we have made it a rule to obtain the support of radiologists and vascular surgeons in operations related to misplaced implants, as well as in preoperative decision making.

As a result of the development of new techniques in spine surgery, more varieties of spinal diseases receive surgical treatment today. Such developments, however, push us toward more difficult challenges, which may increase the chance of facing catastrophic complications. Regarding a cage insertion, we recently tried to position the cage more anteriorly to obtain better lumbar lordosis and sagittal alignment, which may increase the risk of misplacement. We should not only reflect upon our own experience, but also actively try to absorb knowledge from the experience of other surgeons, and make every possible effort to avoid any tragedy. 